\title{
Estimation of early life endogenous surfactant pool and CPAP failure in preterm neonates with RDS
}

\author{
Roberto Raschetti ${ }^{1 \dagger}$, Roberta Centorrino ${ }^{1 \dagger}$, Emmanuelle Letamendia ${ }^{1,2,3}$, Alexandra Benachi ${ }^{2}$, \\ Anne Marfaing-Koka ${ }^{3}$ and Daniele De Luca ${ }^{1,4^{*}}$ (D)
}

\begin{abstract}
Background: It is not known if the endogenous surfactant pool available early in life is associated with the RDS clinical course in preterm neonates treated with CPAP. We aim to clarify the clinical factors affecting surfactant pool in preterm neonates and study its association with CPAP failure.

Methods: Prospective, pragmatic, blind, cohort study. Gastric aspirates were obtained (within the first $6 \mathrm{~h}$ of life and before the first feeding) from 125 preterm neonates with RDS. Surfactant pool was measured by postnatal automated lamellar body count based on impedancemetry, without any pre-analytical treatment. A formal respiratory care protocol based on European guidelines was applied. Clinical data and perinatal risk factors influencing RDS severity or lamellar body count were real-time recorded. Investigators performing lamellar body count were blind to the clinical data and LBC was not used in clinical practice.

Results: Multivariate analysis showed gestational age to be the only factor significantly associated with lamellar body count (standardized $3: 0.233 ; p=0.023$ ). Lamellar body count was significantly higher in neonates with CPAP

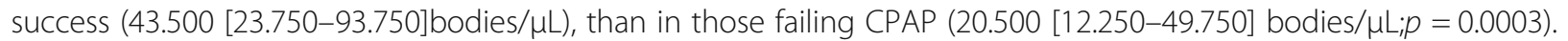
LBC had a moderate reliability to detect CPAP failure (AUC: $0.703(0.615-0.781) ; p<0.0001$; best cut-off: $\leq 30,000$ bodies/ $\mu \mathrm{L}$ ). Upon adjustment for possible confounders, neither lamellar body count, nor its interaction factor with gestational age resulted associated with CPAP failure.
\end{abstract}

Conclusions: Early postnatal lamellar body count on gastric aspirates in CPAP-treated preterm neonates with RDS is significantly influenced only by gestational age. Lamellar bodies are not associated with CPAP failure. Thus, the endogenous surfactant pool available early in life only has a moderate reliability to predict CPAP failure.

Keywords: Lamellar bodies, Preterm neonate, Respiratory distress syndrome, Continuous positive airway pressure

\section{Background}

Antenatal corticosteroid prophylaxis and early application of continuous positive airway pressure (CPAP) are considered the gold standard for, respectively, the prevention and treatment of respiratory distress syndrome (RDS) in preterm neonates [1,2]. Surfactant replacement

\footnotetext{
* Correspondence: daniele.deluca@aphp.fr

${ }^{\dagger}$ Roberto Raschetti and Roberta Centorrino have contributed equally and both are to be considered as first authors

"Division of Pediatrics and Neonatal Critical Care, Medical Center "A.Béclère", South Paris University Hospitals, AP-HP, Paris, France

${ }^{4}$ Physiopathology and Therapeutic Innovation Unit-UNSERM U999, South Paris-Saclay University, Paris, France

Full list of author information is available at the end of the article
}

is recommended when CPAP fails $[3,4]$ and, if performed within the first $3 \mathrm{~h}$ of life, reduces death and/or bronchopulmonary dysplasia [5]. Surfactant is commonly administered based on inspired oxygen fraction $\left(\mathrm{FiO}_{2}\right)$ [3], however, the suggested $\mathrm{FiO}_{2}$ thresholds are arbitrary, they can be reached beyond the optimal 3 h-time window and they lack of any physiopathological background. Moreover, contemporaneous measurement of oxygen saturation or $\mathrm{PaO}_{2}$ might be needed to accurately describe oxygenation. The CPAP level may also influence oxygenation, through its effect on the lung aeration. In fact, both recent pediatric and neonatal

(c) The Author(s). 2019 Open Access This article is distributed under the terms of the Creative Commons Attribution 4.0 International License (http://creativecommons.org/licenses/by/4.0/), which permits unrestricted use, distribution, and 
acute respiratory distress syndrome (ARDS) definitions include these variables in appropriate indexes $[6,7]$.

The available endogenous surfactant also influences lung aeration and, therefore, assessing the surfactant pool available early in life could be interesting to better understand the clinical picture and eventually guide surfactant replacement. Lamellar bodies are intracellular, easily measurable, surfactant storage granules released from type II-pneumocytes [8,9]. Once in the alveolar space, lamellar bodies are subjected to the breathing cycle and form the surfactant layer adsorbed at the gas/ liquid interface $[9,10]$. Thus, lamellar body count (LBC) is an estimation of the available endogenous surfactant. LBC correlates with lung aeration evaluated by a semi-quantitative lung ultrasound score $[11,12]$. LBC can be realized on amniotic fluid and is used as prenatal lung maturity test, as it has a good reliability to predict the RDS occurrence [13, 14], but is also doable postnatally (at the delivery or using gastric aspirates obtained before the first feeding) [15-17].

Despite this physiopathological background, the link between the available surfactant pool and the CPAP failure has never been investigated. Our purposes are: 1) to clarify what are the clinical factors affecting early postnatal LBC in CPAP-treated preterm neonates with RDS; and 2) to investigate reliability of postnatal $L B C$ and its association to CPAP failure.

\section{Methods}

\section{Patients}

We designed a pragmatic, observational, blind, prospective cohort study. The study was conducted during 2014-2015 in an academic tertiary referral neonatal intensive care unit (NICU). All inborn preterm neonates ( $<37$ weeks' gestation) with RDS treated with nasal CPAP were eligible for the study. In detail, RDS was diagnosed when the following criteria [6] were met: 1) occurrence of respiratory distress within the first $24 \mathrm{~h}$ of life; 2) presence of typical lung ultrasound or chest X-rays findings; [18, 19] 3) complete, sustained and prompt oxygenation improvement after surfactant replacement or significant improvement under CPAP which prevented surfactant administration; 4) no other respiratory disorders, as detailed below.

Exclusion criteria were: 1) chromosomal abnormalities or complex congenital malformations; 2) congenital lung diseases; 3) need for surgery in the first week of life;

4) early onset severe sepsis/septic shock, as defined elsewhere; [20] 5) transient tachypnea of the neonate, defined as mild (Silverman score $\leq 3$ ) respiratory distress occurring in a neonate of more than 34 weeks' gestation presenting with typical lung ultrasound or chest X-rays findings and resolving within the first $72 \mathrm{~h}$ of life; $[6,18$, 19] 6) meconium aspiration syndrome, defined as the presence of meconium-stained amniotic fluid and airway secretions, occurrence of respiratory failure early from birth and typical lung imaging; [21] 7) blood aspiration syndrome, defined as respiratory distress and bloodstained amniotic fluid and airway secretions, onset of respiratory failure early from birth and typical lung imaging; [22] 8) pulmonary haemorrhage, defined as respiratory distress suddenly occurring together with bright blood-stained airway secretions, presence of left-to-right ductal shunting and typical lung imaging; [23] 9) any kind of neonatal ARDS, defined according to criteria described earlier [6].

All pregnancies received full prenatal care: gestational age was estimated based on the postmenstrual date and early gestation ultrasound findings; antenatal betamethasone was administered as two 12-mg doses $24 \mathrm{~h}$ apart, whenever delivery was expected to occur before 34 weeks. Steroid prophylaxis was considered complete when the second dose was given at least $24 \mathrm{~h}$ before the delivery. Small for gestational age (SGA) babies were classified according to Fenton's curves [24]. Chorioamnionitis and obstetric cholestasis were clinically defined as described elsewhere [25, 26]. Gestational diabetes and pre-eclampsia were diagnosed as per current international guidelines $[27,28]$.

\section{Respiratory protocol}

We use a formal respiratory care protocol derived from European guidelines for RDS management [3]. Delivery room intubation was performed only on persistently apnoeic or bradycardic babies, unresponsive to face-mask ventilation, according to international guidelines on neonatal resuscitation [29]. Neonates are started with variable or continuous flow-CPAP if they were $\leq$ or $>32$ weeks' gestation, respectively. This choice aims to reduce the work of breathing as much as possible in the smallest preterm neonates [30]. Appropriately sized nasal masks were used and particular care was applied to minimize CPAP interruptions. CPAP was set at 6 $\mathrm{cmH}_{2} \mathrm{O}$ and oxygen was added only if CPAP was not enough to maintain peripheral oxygen saturation within 90 and $95 \%$ [31]. Pacifiers of adequate size with drops of $30 \%$ glucose solution were used to reduce leaks and provide sedation, if needed [31]. CPAP was started in the delivery room just after stabilization or upon NICU admission for babies with gestational age $\leq$ or $>32$ weeks, respectively [12].

CPAP failure was defined as need for surfactant replacement to treat RDS in the first $48 \mathrm{~h}$ of life. Surfactant was administered as poractant- $\alpha$ (Chiesi Farmaceutici, Parma, Italy; $200 \mathrm{mg} / \mathrm{Kg}$ ), whenever $\mathrm{FiO}_{2}$ exceeded 0.3 or 0.4 for babies with gestational age $\leq$ or $>28$ weeks' gestation, respectively. The technique for surfactant administration, ventilation policy and use of sedation or 
caffeine have been described earlier [32]. All new residents and fellows are trained about the respiratory protocol every 6 months.

\section{Lamellar bodies measurement}

We have chosen to use gastric aspirates, as this sampling is practically easier to perform than the amniotic fluid collection at the delivery. Moreover, specimens obtained through gastric aspirates are less likely to be contaminated by blood or meconium. Samples were collected upon the appearance of respiratory distress, within the first $3 \mathrm{~h}$ of life and anyway before the first feeding using a soft, end-hole suction catheter and applying a negative pressure of $-100 \mathrm{mmHg}$. Samples were collected in sterile polypropylene traps, immediately sent to the laboratory at room temperature and divided into two identical aliquots. The first aliquot was used for routine microbiological test and the second for LBC. We wanted to perform a pragmatic study in a large-volume perinatal centre, thus samples were not processed adding dithiothreitol or any other solvents, nor were they centrifuged or frozen. Samples were directly aspirated by automated cell counters (LH780, Beckman Coulter, Brea, CA USA) and lamellar bodies were counted by impedancemetry through the platelet channel of the analyser, as previously described [9]. When the fluid was too viscous it was diluted with isotonic saline $(1: 1 \mathrm{v} / \mathrm{v})$ and results were corrected for dilution. Investigators performing LBC were blinded to patients' clinical conditions.

\section{Data collection}

Basic clinical data and perinatal risk factors influencing the clinical severity of RDS or known to affect antenatal $\mathrm{LBC}$ were recorded in real time. $\mathrm{LBC}$ was recorded in a specific spreadsheet and merged with clinical data only at the end of recruitment, thus clinicians were blinded to $\mathrm{LBC}$ and they were not used for any clinical decision. Relevant NICU protocols did not change during the study. Participation to the study did not modify the common clinical care, since gastric aspirates culture is routinely used as microbiological screening in NICU-admitted babies (if obtained before the first feeding), according to our routine clinical protocol. The study was approved by local institutional review board (n.13-046) and consent was obtained from parents or guardian upon NICU admission.

\section{Statistics}

Since this was the first study about LBC and CPAP failure, a formal sample size calculation was unfeasible: we continued the recruitment until we reached a sample size similar to that of other studies using the same technique [14]. Population was divided in the CPAP success or failure cohorts. Continuous data were tested for normality with Kolmogorov-Smirnov test, expressed as mean (standard deviation) or median [interquartile range] and then contrasted with Student or Mann-Whitney, accordingly. Spearman correlation was also performed between $\mathrm{LBC}$ and continuous variables. Proportions were compared with $\chi^{2}$ or Fisher test, as appropriate.

Multiple linear regression was finally applied on the whole population to identify factors affecting LBC. All variables potentially able to influence $\mathrm{LBC}$ were used as covariates. In detail, covariates included in the model were gestational age, antenatal steroids, clinical chorioamnionitis, obstetric cholestasis, 5'Apgar score and SGA status. In order to avoid multicollinearity, birth weight was not used, since it is highly correlated with gestational age $(r h o=0.844 ; p<0.0001)$. Enter technique was used and no covariates selection method was applied.

Receiver operating characteristic (ROC) analysis was used to evaluate the reliability of LBC to predict CPAP failure: area under the curve (AUC) and reliability data were reported with ( $95 \%$ confidence intervals). The relationship between LBC and CPAP failure was studied adjusting for possible confounders using a Poisson regression, with a logit link function and adjusted relative risks $\left({ }_{\mathrm{a}} \mathrm{RR}\right)$ were calculated. Gestational age, antenatal steroids, pre-eclampsia and LBC were inserted as covariates, since they had a $p<0.2$ at the univariate comparison between CPAP success and failure cohorts. An alternative model was also tested including an interaction term between LBC and gestational age, as both these variables are significantly associated to RDS occurrence [33]. In order to avoid multicollinearity, birth weight was not used, since it is highly correlated with gestational age and SGA babies were not different between the two cohorts. Model goodness-of-fit was evaluated by Omnibus test. All analyses were performed with SPSS Version 15.0 (SPSS Inc., Chicago, IL, USA) and Medcalc 13.3 (MedCalc bvba, Ostend, Belgium). $p$-values $<0.05$ were considered statistically significant.

\section{Results}

We enrolled 192 neonates but in 67 LBC was not technically feasible due to the fluid viscosity $(n=50)$, meconium or blood contamination $(n=6)$ or insufficient volume $(n=11)$. Basic details and perinatal risk factors for RDS for the 125 neonates enrolled are described in Table 1: patients failing CPAP had lower gestational age, birth weight and tended to receive less antenatal steroids. CPAP failure occurred at 2.5 [1.5-4] h of life always appearing as increasing oxygen requirement and dyspnoea. No mother had hypothyroidism; no pneumothorax or pneumomediastinum were observed. Gastric aspirates were collected at $1[0.5-2] \mathrm{h}$ of life. There were 
Table 1 Basic population details and perinatal factors influencing RDS severity

\begin{tabular}{|c|c|c|c|c|}
\hline Patients & Whole population (125) & CPAP success (85) & CPAP failure (40) & $\mathrm{p}$ \\
\hline Gestational age (weeks) & $31.6(3.3)$ & $32.9(2.4)$ & $29.3(3.2)$ & $<0.0001$ \\
\hline Birth weight (grams) & $1641(648)$ & $1807(593)$ & $1327(641)$ & $<0.0001$ \\
\hline Complete antenatal steroids & $89(71 \%)$ & $56(66 \%)$ & $24(60 \%)$ & 0.09 \\
\hline Clinical chorioamnionitis & $7(11 \%)$ & $3(3.5 \%)$ & $4(10 \%)$ & 0.211 \\
\hline Gestational diabetes & $2(1.6 \%)$ & $2(2.3 \%)$ & 0 & 0.999 \\
\hline Obstetric cholestasis & $8(6 \%)$ & $6(7 \%)$ & $2(5 \%)$ & 0.962 \\
\hline Pre-eclampsia & $21(17 \%)$ & $17(20 \%)$ & $4(10 \%)$ & 0.155 \\
\hline Premature rupture of membranes & $10(8 \%)$ & $8(9.4 \%)$ & $2(5 \%)$ & 0.500 \\
\hline Caesarean section & $71(57 \%)$ & $67(79 \%)$ & $33(82 \%)$ & 0.811 \\
\hline 5' Apgar score & $8.9(1.3)$ & $8.9(1.1)$ & $8.8(1.7)$ & 0.529 \\
\hline SGA neonates & $18(14 \%)$ & $13(15 \%)$ & $5(12 \%)$ & 0.660 \\
\hline
\end{tabular}

Data are expressed as mean (standard deviation) or number (\%). $p$-values refer to the comparison between CPAP success and failure cohorts with Student, $x^{2}$ or Fisher exact test, as appropriate

no differences between neonates enrolled in the study and those for whom LBC was unfeasible for technical reasons (gestational age: $33.3(2.9)$ weeks, $p=0.270$; 5'Apgar: 9 (1.1), $p=0.593$; antenatal steroids 61.2\%, $p=$ 0.195 ; caesarean delivery $56.7 \%, p=0.999$; SGA neonates: $6 \%, p=0.10$; CPAP failure: $28.4 \%, p=0.627)$ ).

LBC was significantly higher in patients who received a complete course of prenatal steroids (38.000 [19.200$76.500] \mathrm{LBC} / \mu \mathrm{L}$ ), than in those who received an incomplete course or no prophylaxis 23.000 [12.500-44.200]
$\mathrm{LBC} / \mu \mathrm{L} ; p=0.032)$, by the univariate analysis. LBC significantly correlated with gestational age (rho = $0.331 ; p<0.001$ ) and birth weight ( $r h o=0.844 ; p<0.001$ ) LBC did not differ between neonates born to mothers with or without pre-eclampsia (38.000 [12.000-96.500] vs 33.000 [18.000-69.000] LBC/ $\mu \mathrm{L} ; p=0.764$ ), clinical chorioamnionitis $(25.000 \quad[9000-45.000]$ vs 35.000 $[18.500-74.000] \mathrm{LBC} / \mu \mathrm{L} ; p=0.171)$ or obstetric cholestasis $(52.500$ [10.500-69.750] vs 33.500 [18.250-71.500] $\mathrm{LBC} / \mu \mathrm{L} ; p=0.764)$ and between neonates small or

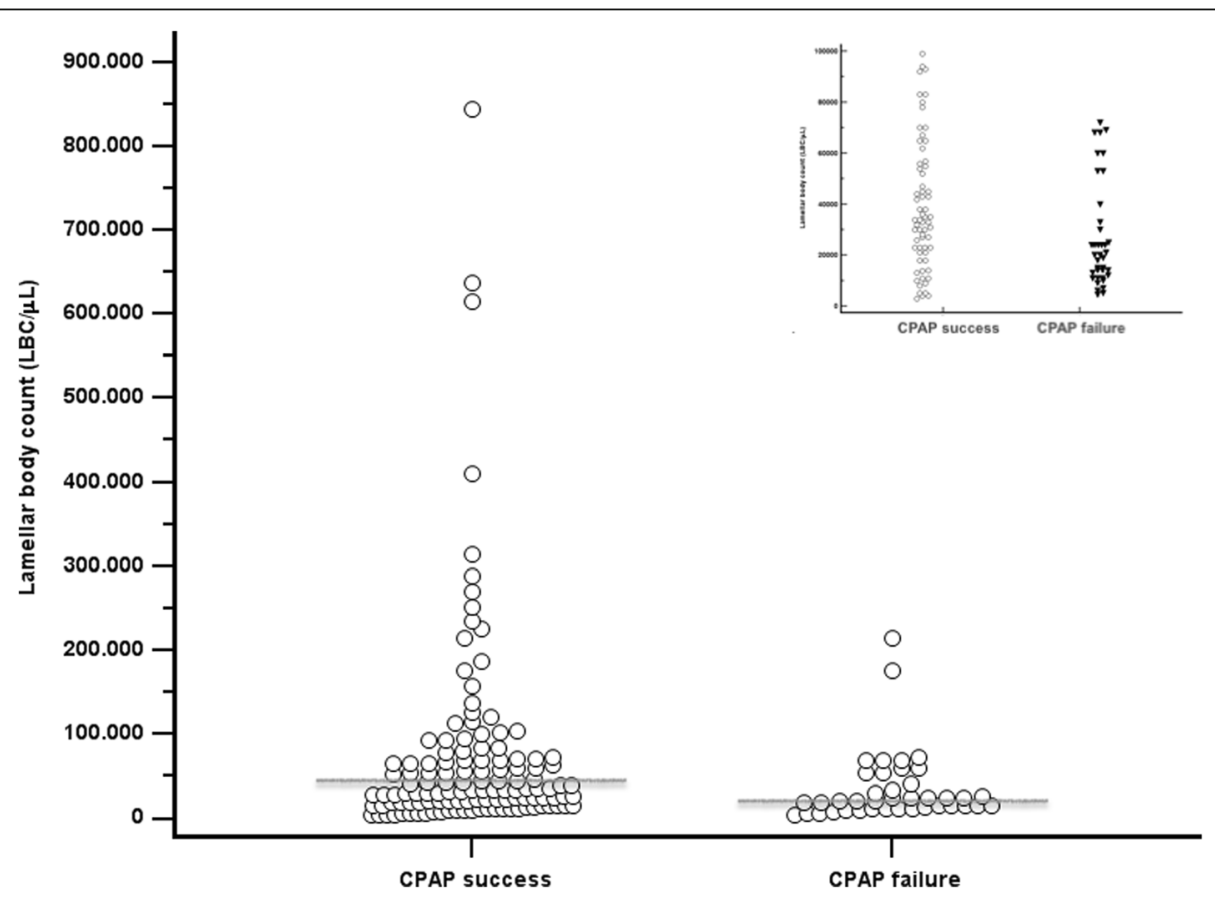

Fig. 1 Lamellar body count (LBC) in CPAP success and failure cohorts. Data from all patients are depicted in the big picture, while the insert illustrates only data from patients with lamellar bodies are $<100,000 \mu \mathrm{L}$. Grey horizontal lines represent median values. LBC is higher in the CPAP success than in CPAP failure cohort ( $p=0.0003$; Mann-Whitney test); full data are reported in the text 
appropriate for gestational age (40.500 [26.250-134.250] vs 33.000 [15.000-68.250] LBC/ $\mu \mathrm{L} ; p=0.169)$. LBC $\mathrm{did}$ not correlate with 5'Apgar score $(r h o=0.024 ; p=809)$. Multivariate analysis showed gestational age to be the only factor significantly associated with LBC (standardized $\beta: 0.214 ; p=0.034$ ); all other covariates were removed from the model.

Figure 1 shows that LBC was significantly higher in neonates with CPAP success $(43.500$ [23.75093.750]LBC/ $\mu \mathrm{L})$, than in those failing CPAP $(20.500$ [12.250-49.750] LBC/ $\mu \mathrm{L} ; p=0.0003)$. Subgroup analysis showed that neonates of gestational age $\leq 32$ weeks with CPAP success $(38,000[22000-102,000] \mathrm{LBC} / \mu \mathrm{L})$ had significantly higher LBC than those failing CPAP $(20,000$ $[12000-30,000] \mathrm{LBC} / \mu \mathrm{L} ; p=0.008)$. Conversely, subgroup analysis for babies aged more than 32 weeks showed no significant differences $(53,000$ [18730$117,500]$ vs 45,000 [27000-92,000] LBC/ $\mu \mathrm{L} ; p=0.845)$.

ROC analysis is depicted in Fig. 2. LBC had a moderate reliability to detect CPAP failure (AUC: 0.703 $(0.615-0.781) ; p<0.0001$; best cut-off: $\leq 30,000 \mathrm{LBC} / \mu \mathrm{L}$; sensitivity: 70 (53.5-83.4)\%; specificity: 67.1 (56-76.9)\%; positive likelihood ratio: 2.1 (1.5-3.1); negative likelihood ratio: $0.45(0.3-0.7)$; positive predictive value: 50 (36.3$67.3) \%$; negative predictive value: 82.6 (71.6-90.7)\%). Reliability of $\mathrm{LBC}$ was not improved when performing a subgroup analysis for neonates of $\leq 32$ weeks (AUC:
$0.314(0.181-0.447))$ or $>32$ weeks gestation (AUC: $0.473(0.210-0.735))$.

Upon adjustment for possible confounders (Table 2), gestational age and prenatal steroid prophylaxis resulted associated to CPAP failure, while LBC and the interaction factor ' $\mathrm{LBC}$ "gestational age' did not.

\section{Discussion}

The knowledge of the available endogenous surfactant pool in RDS neonates undergoing CPAP might be an important information to understand the physiopathology and the clinical picture of each case. Moreover, if a point-of-care test measuring surfactant pool would be able to predict CPAP failure, that could theoretically help a more tailored and optimized surfactant therapy. We measured the early life surfactant pool in preterm neonates who received optimal perinatal care and were treated with CPAP according to formal protocols based on more recent evidences [1-3].

Our study shows that, in such patients, postnatal automated LBC (without any pre-analytical step): 1) is significantly influenced by gestational age, while other clinical factors do not seem to be relevant; 2) is not significantly associated with CPAP failure. Thus, the endogenous surfactant pool available early in life only has a moderate reliability to predict CPAP failure at any gestational age.

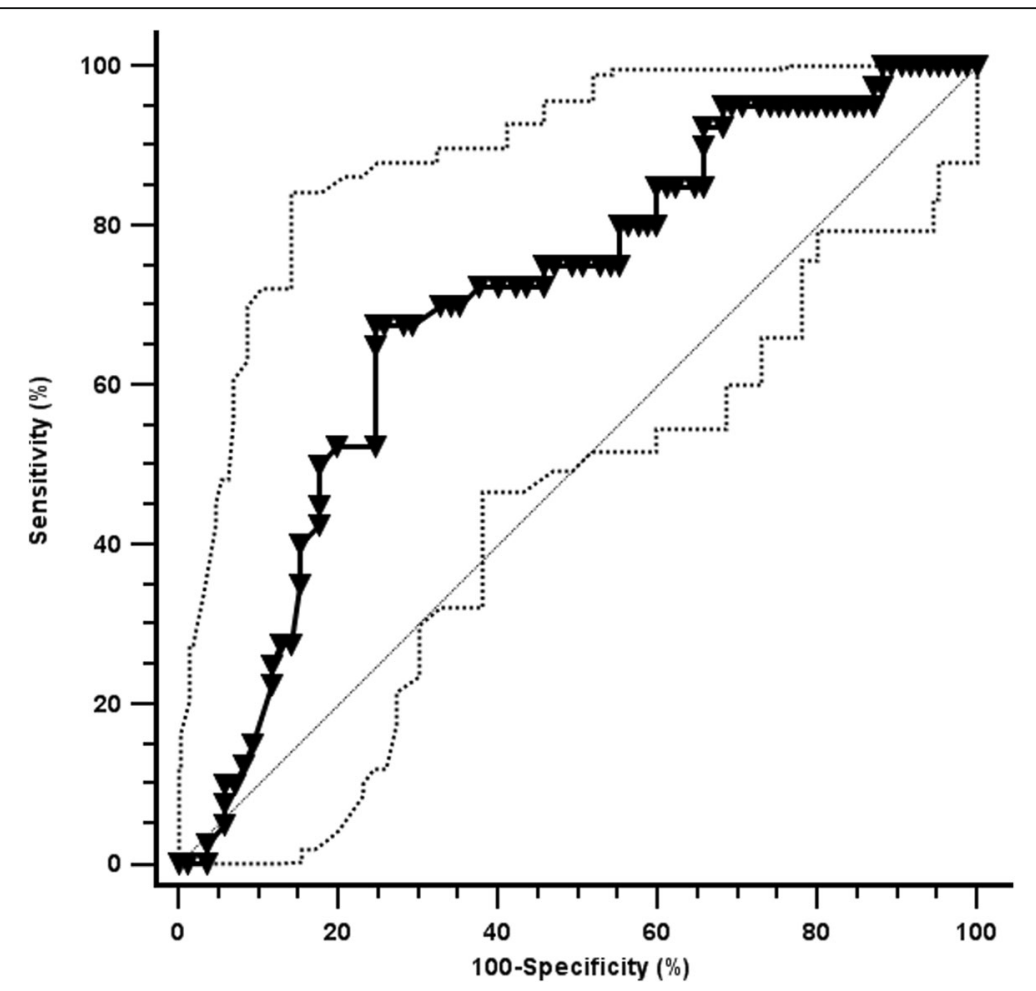

Fig. 2 Receiver Operator Characteristic curve for the prediction of CPAP failure. Black and hatched lines represent the area under the curve (AUC) and its $95 \%$ confidence interval. Diagonal line represents an AUC of 0.5. More details in the text 
Table 2 Multivariate analysis results: factors associated with CPAP failure

\begin{tabular}{|c|c|c|}
\hline & ${ }_{\mathrm{a}} \mathrm{RR}$ for CPAP failure $(95 \% \mathrm{Cl})$ & $p$ \\
\hline \multicolumn{3}{|l|}{ 1st model } \\
\hline Gestational age & $0.62(0.51-0.76)$ & $<0.0001$ \\
\hline Incomplete or no prenatal steroids & $3.2(1.07-9.6)$ & 0.037 \\
\hline Pre-eclampsia & $3(0.7-12.2)$ & 0.123 \\
\hline LBC & $0.99(0.9-1.004)$ & 0.218 \\
\hline \multicolumn{3}{|l|}{ 2nd model (with interaction term) } \\
\hline LBC ${ }^{*}$ Gestational age & $1.001(0.99-1.003)$ & 0.213 \\
\hline
\end{tabular}

These two findings are novel as, to the best of our knowledge, LBC had never been tested in these conditions or aiming to predict CPAP failure in neonates with established RDS, while LBC has been used to predict RDS occurrence [14, 17, 33]. Our results might seem conflicting with experimental data showing increased LBC in lung tissue of animals treated with prenatal steroids. Nevertheless, we enrolled neonates with already established RDS according to clinical and imaging integrated data: thus, in our population, RDS occurred despite the eventual prenatal steroid prophylaxis, which did not produce a complete benefit. It is conceivable that, under these conditions, gestational age would be the only remaining variable significantly influencing LBC, since steroids failed to boost surfactant production enough to prevent RDS insurgence.

About the lack of LBC reliability to predict CPAP failure, ROC analysis suggests as best cut-off value the same $(30.000 \mathrm{LBC} / \mu \mathrm{L})$ that has been indicated to predict RDS occurrence, [14] although the reliability is much lower in our study. This may be related to the effect of CPAP. CPAP is known to be efficacious to treat RDS and spare surfactant replacement in preterm neonates [2]. The mean time needed for preterm neonates to produce enough endogenous surfactant is approximately 4 days [34] and meanwhile CPAP keeps the alveoli open improving the oxygenation, despite the relative surfactant deficiency. LBC only gives a picture of the surfactant pool available early after birth. Thus, the occurrence of RDS is more closely related to the initially available endogenous surfactant (that is, to LBC), than the clinical evolution overtime, which also depends on surfactant production along the days and to CPAP-induced alveolar recruitment. In fact, phosphatidylcholine concentration in fetal lung fluid does not correlate with clinical severity of RDS in preterm lambs [35]. Other factors influencing the clinical severity of RDS overtime (such as the degrees of alveolarization and lung tissue inflammation, type and level of CPAP, amount of extravascular lung water, nursing experience) may also have contributed to the reduced LBC reliability [36]. Multivariate analysis confirmed this issue, since the endogenous available surfactant pool, as measured by $\mathrm{LBC}$, is not significantly associated to CPAP failure. This is fully consistent with the explanations suggested above, as the surfactant pool might impact on the RDS occurrence more than on its evolution [13, 14]. Conversely, modern perinatal care is likely to significantly impact on the course of RDS, as early CPAP is able to avoid surfactant treatment in a significant proportion of patients [37]. Subgroup analysis also confirms the relative lack of LBC reliability to predict CPAP failure at any gestational age and does not seem to suggest a particular patient subgroups where the technique could be more useful.

If we compare LBC to two other recently proposed tools to predict CPAP failure, such as the lung ultrasound score [12,38] and the surfactant adsorption test (SAT), [11] in both cases LBC results inferior in terms of AUC. This can have several explanations. First, LBC is performed early from birth and CPAP failure may occur significantly later: a test performed significantly earlier tends to be less accurate than a test performed closer to the outcome to predict. Moreover, lung ultrasound score describes the lung aeration rather than the surfactant pool available, thus it takes into consideration the CPAP-induced alveolar recruitment and can also be serially repeated overtime. SAT is performed in the first hours of life similar to LBC, but it describes surfactant quality and properties, rather than its available amount. In fact, SAT measures surfactant adsorption and accumulation overtime at the air/liquid interface, for a given amount of phospholipids [11]. While the amount of available surfactant may be relevant for the RDS occurance, its quality and biophysical properties could be more important for the clinical severity, once RDS is established. In fact, surfactant adsorption significantly depends on surfactant-protein B, which is known to be related to the clinical severity of RDS [39].

We acknowledge some study limitations. More samples could have been assayed introducing additional analytical steps, such as solvent dilution or centrifugation/ vortexing [16]. However, these steps could theoretically 
increase complexity and make the test less immediately available. Therefore, automated LBC without any pre-analytical steps might not be always pragmatically suitable. We aimed to investigate surfactant pool as estimated by automated postnatal LBC on gastric aspirates without any particular evaluation of surfactant activity or composition. LBC might vary with the relative proportion of efflux of fetal lung fluid mixing with swallowed amniotic fluid and gastric secretions and this could lead to a relative underestimation. Also, it may be difficult to obtain gastric aspirates early from birth for logistic reasons and the postnatal age may affect the reliability of gastric aspirates in representing lung fluid. Therefore, the proportion of fetal lung fluid in gastric aspirates may be influenced by several factors, including clearance/dilution of gastric fluid. Thus, gastric aspirates samples may be biased regarding quantity and quality of surfactant, and these specimens might not equate with the surfactant pool available in the lung. However, we feel that our results are interesting, since they come from a pragmatic study reflecting NICU real life. The pragmatic approach is particularly appropriate for tools or interventions representing refinements of current clinical care [40]. Thus, we used a quick assay to estimate surfactant pool, in a homogeneous population of preterm babies with RDS without any other respiratory disorders and subjected to good perinatal care and a formal, modern, respiratory care protocol. This is representative of the usual neonatal care in developed countries. We cannot evaluate the effect of maternal diabetes or hypothyroidism, as few or no cases presented with these disorders. Nonetheless, we have been able to evaluate all other major clinical factors influencing endogenous surfactant pool and clinical picture of RDS in preterm neonates. It would have been interesting to deeply study the reliability of LBC in different classes of gestational age and, particularly, in extremely preterm neonates who are more likely to need a "guided" surfactant therapy. However, we did not have a large enough population and a preliminary subgroup analysis did not seem to show any difference. This analysis could have been biased and may need to be repeated in larger populations. However, the availability of more accurate tools (such as semiquantitative lung ultrasound and the surfactant adsorption or microbubble tests) reduces the interest in this regard [11, 12, 38, 41]. Finally, ours is a relatively small population but comparable to those of other studies in the field [14].

\section{Conclusion}

Early life LBC on gastric aspirates in CPAP-treated preterm neonates with RDS is significantly influenced by gestational age. LBC is not associated with CPAP failure. Thus, the endogenous surfactant pool available early in life only has a moderate reliability to predict CPAP failure. Further studies are needed to better clarify the biological factors associated to CPAP failure in similar populations.

\begin{abstract}
Abbreviations
${ }_{a}$ RR: Adjusted relative risks; AUC: Area under the curve; CPAP: Continuous positive airway pressure; $\mathrm{FiO}_{2}$ : Inspired oxygen fraction; LBC: Lamellar body count; NICU: Neonatal intensive care unit; RDS: Respiratory distress syndrome; ROC: Receiver operating characteristic; SGA: Small for gestational age
\end{abstract}

\section{Acknowledgments}

Not applicable

Funding

None

\section{Availability of data and materials}

The datasets used and/or analysed during the current study are available from the corresponding author on reasonable request.

\section{Authors' contributions}

$\mathrm{RR}$ and RC, prepared the databases and collected all the data, coordinated the sample transfer, drafted the initial manuscript, they contributed equally to and reviewed and revised the manuscript. $E L, A B$ and $A M K$ designed the data and sample collection procedures, analysed samples and reviewed and revised the manuscript. DDL conceptualized and designed the study, coordinated and supervised it, performed the statistical analysis, and critically reviewed the manuscript for important intellectual content. All authors approved the final manuscript as submitted and agree to be accountable for all aspects of the work.

\section{Ethics approval and consent to participate}

This study is a part of a project on surfactant and respiratory support in neonates approved by the Ethical Committee at the South Paris University Hospital (n.CPP13-046) and consent to participation was obtained from parents or guardian upon NICU admission.

\section{Consent for publication}

Not applicable

\section{Competing interests}

DDL has received grants for research and educational projects from Chiesi Pharmaceuticals spa and ABBVIE inc. He also received travel grants from ABBVIE inc. He served as consultant and lecturer for both Chiesi

Pharmaceuticals spa and ABBVIE inc. Finally, he has been the Chair of the external advisory board for Chiesi Pharmaceuticals spa and a member of the ABBVIE inc. These companies produce two surfactants, but they had no role in design, preparation, review, approval of the manuscript or decision to submit it for publication. The other authors have no conflicts of interest to disclose.

\section{Publisher's Note}

Springer Nature remains neutral with regard to jurisdictional claims in published maps and institutional affiliations.

\section{Author details}

1Division of Pediatrics and Neonatal Critical Care, Medical Center "A.Béclère", South Paris University Hospitals, AP-HP, Paris, France. ${ }^{2}$ Division of Obstetrics and Gynecology, Medical Center "A.Béclère", South Paris University Hospitals, AP-HP, Paris, France. 'Division of Hematology, Medical Center "A.Béclère", South Paris University Hospitals, AP-HP, Paris, France. ${ }^{4}$ Physiopathology and Therapeutic Innovation Unit-UNSERM U999, South Paris-Saclay University, Paris, France. 
Received: 15 January 2019 Accepted: 2 April 2019 Published online: 16 April 2019

\section{References}

1. Roberts D, Brown J, Medley N, Dalziel SR. Antenatal corticosteroids for accelerating fetal lung maturation for women at risk of preterm birth. Cochrane Pregnancy and Childbirth Group, editor. Cochrane Database Syst Rev [Internet]. 2017 [cited 2018 May 25]; Available from: http://doi.wiley. com/https://doi.org/10.1002/14651858.CD004454.pub3

2. Subramaniam P, Ho JJ, Davis PG. Prophylactic nasal continuous positive airway pressure for preventing morbidity and mortality in very preterm infants. Cochrane Neonatal Group, editor. Cochrane Database Syst Rev [Internet]. 2016 [cited 2018 May 25]; Available from: http://doi.wiley.com/ https://doi.org/10.1002/14651858.CD001243.pub3

3. Sweet DG, Carnielli V, Greisen G, Hallman M, Ozek E, Plavka R, et al. European consensus guidelines on the Management of Respiratory Distress Syndrome - 2016 update. Neonatology. 2017;111:107-25.

4. American Academy of Pediatrics. Committe on Fetus and Newborn. Respiratory support in preterm infants at birth. Pediatrics. 2014;133:171-4.

5. Bahadue FL, Soll R. Early versus delayed selective surfactant treatment for neonatal respiratory distress syndrome. Cochrane Database Syst Rev. 2012 Nov 14;11:CD001456. https://doi.org/10.1002/14651858.CD001456.pub2.

6. De Luca D, van Kaam AH, Tingay DG, Courtney SE, Danhaive O, Carnielli VP, et al. The Montreux definition of neonatal ARDS: biological and clinical background behind the description of a new entity. Lancet Respir Med. 2017:5:657-66

7. Khemani RG, Smith LS, Zimmerman JJ, Erickson S. Pediatric acute respiratory distress syndrome: definition, incidence, and epidemiology. Pediatr Crit Care Med. 2015;16:S23-40.

8. Dietl P, Haller T. Exocytosis of lung surfactant: from the secretory vesicle to the air-liquid Interface. Annu Rev Physiol. 2005;67:595-621.

9. Dubin SB. Characterization of amniotic fluid lamellar bodies by resistivepulse counting: relationship to measures of fetal lung maturity. Clin Chem. 1989:35:612-6.

10. Danhaive $\mathrm{O}$, Chapin C, Horneman H, Cogo PE, Ballard PL. Surface film formation in vitro by infant and therapeutic surfactants: role of surfactant protein B. Pediatr Res. 2015;77:340-6.

11. Autilio C, Echaide M, Benachi A, Marfaing-Koka A, Capoluongo ED, Pérez-Gil J, et al. A noninvasive surfactant adsorption test predicting the need for surfactant therapy in preterm infants treated with continuous positive airway pressure. J Pediatr. 2017;182:66-73.e1.

12. Brat R, Yousef N, Klifa R, Reynaud S, Shankar Aguilera S, De Luca D. Lung ultrasonography score to evaluate oxygenation and surfactant need in neonates treated with continuous positive airway pressure. JAMA Pediatr. 2015;169:e151797.

13. Neerhof MG, Dohnal JC, Ashwood ER, Lee IS, Anceschi MM. Lamellar body counts: a consensus on protocol. Obstet Gynecol. 2001;97:318-20.

14. Besnard AE, Wirjosoekarto SAM, Broeze KA, Opmeer BC, Mol BWJ. Lecithin/ sphingomyelin ratio and lamellar body count for fetal lung maturity: a meta-analysis. Eur J Obstet Gynecol Reprod Biol. 2013;169:177-83.

15. Wijnberger LDE, de Kleine M, Voorbij HAM, Arabin B, Engel H, Bruinse HW, et al. Comparison of vaginal and transabdominal collection of amniotic fluid for fetal lung maturity tests. J Matern Fetal Neonatal Med. 2010;23:613-6.

16. Machado LU, Fiori HH, Baldisserotto M, Ramos Garcia PC, Vieira ACG, Fiori RM. Surfactant deficiency in transient tachypnea of the newborn. J Pediatr. 2011;159:750-4

17. Verder H, Ebbesen F, Brandt J, Dahl M, Esberg G, Eschen C, et al. Lamellar body counts on gastric aspirates for prediction of respiratory distress syndrome. Acta Paediatr Oslo Nor 1992. 2011;100:175-80.

18. Raimondi F, Yousef N, Migliaro F, Capasso L, De Luca D. Point-of-care lung ultrasound in neonatology: classification into descriptive and functional applications. Pediatr Res. 2018. https://doi.org/10.1038/s41390-018-0114-9. [Epub ahead of print].

19. Arthur R. The neonatal chest X-ray. Paediatr Respir Rev. 2001;2:311-23.

20. Goldstein B, Giroir B, Randolph A. International pediatric sepsis consensus conference: definitions for sepsis and organ dysfunction in pediatrics*. Pediatr Crit Care Med. 2005;6:2-8.

21. Piastra M, Yousef $N$, Brat R, Manzoni P, Mokhtari M, De Luca D. Lung ultrasound findings in meconium aspiration syndrome. Early Hum Dev. 2014;90:S41-3.
22. Celik IH, Demirel G, Canpolat FE, Erdeve O, Dilmen U. Surfactant therapy for maternal blood aspiration: an unusual cause of neonatal respiratory distress syndrome. Indian J Pediatr. 2012;79:1358-9.

23. Ren X-L, Fu W, Liu J, Liu Y, Xia R-M. Lung ultrasonography to diagnose pulmonary hemorrhage of the newborn. J Matern-Fetal Neonatal Med. 2017;30:2601-6.

24. Fenton TR. A new growth chart for preterm babies: Babson and Benda's chart updated with recent data and a new format. BMC Pediatr. 2003;3:13.

25. Been JV, Vanterpool SF, de Rooij JDE, Rours GIJG, Kornelisse RF, van Dongen MCJM, et al. A clinical prediction rule for histological Chorioamnionitis in preterm newborns. Baud O, editor. PLoS One 2012;7:e46217.

26. Zecca E, De Luca D, Marras M, Caruso A, Bernardini T, Romagnoli C. Intrahepatic cholestasis of pregnancy and neonatal respiratory distress syndrome. Pediatrics 2006;117:1669-72.

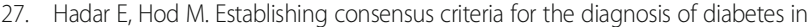
pregnancy following the HAPO study. Ann N Y Acad Sci. 2010;1205:88-93.

28. Preventive Services Task Force US, Bibbins-Domingo K, Grossman DC, Curry SJ, Barry MJ, Davidson KW, et al. Screening for preeclampsia: US Preventive Services Task Force recommendation statement. JAMA. 2017;317:1661-7.

29. Perlman JM, Wyllie J, Kattwinkel J, Wyckoff MH, Aziz K, Guinsburg R, et al. Part 7: neonatal resuscitation: 2015 international consensus on cardiopulmonary resuscitation and emergency cardiovascular care science with treatment recommendations (reprint). Pediatrics. 2015;136:S120-66.

30. Pandit PB, Courtney SE, Pyon KH, Saslow JG, Habib RH. Work of breathing during constant- and variable-flow nasal continuous positive airway pressure in preterm neonates. Pediatrics. 2001;108:682-5.

31. Gizzi C, Klifa R, Pattumelli M, Massenzi L, Taveira M, Shankar-Aguilera S, et al. Continuous positive airway pressure and the burden of Care for Transient Tachypnea of the neonate: retrospective cohort study. Am J Perinatol. 2015; 32:939-43.

32. Dell'Orto V, Bourgeois-Nicolaos N, Rouard C, Romain O, Shankar-Aguilera S, Doucet-Populaire F, et al. Cell count analysis from Nonbronchoscopic Bronchoalveolar lavage in preterm infants. J Pediatr. 2018;200:30-37.e2. https://doi.org/10.1016/j.jpeds.2018.04.074

33. Tsuda H, Hirakawa A, Kotani T, Sumigama S, Mano Y, Nakano T, et al. Risk assessment for neonatal RDS/TTN using gestational age and the amniotic lamellar body count in twin pregnancies. Clin Chim Acta. 2015;451:301-4.

34. Cavicchioli P, Zimmermann LJ, Cogo PE, Badon T, Giordano G, Torresin M, et al. Endogenous surfactant turnover in preterm infants with respiratory distress syndrome studied with stable isotope lipids. Am J Respir Crit Care Med. 2001;163:55-60.

35. Jobe A, Jacobs $H$, Ikegami M. Lack of correlation of severity of lung disease with the phosphatidylcholine concentration in fetal lung fluid from premature lambs at 133-136 days gestational age. J Dev Physiol. 1984;6: 417-21.

36. Jobe $\mathrm{AH}$, Ikegami M, Jacobs HC, Jones SJ. Surfactant pool sizes and severity of respiratory distress syndrome in prematurely delivered lambs. Am Rev Respir Dis. 1983;127:751-5.

37. Wiingreen R, Greisen G, Ebbesen F, Petersen JP, Zachariassen G, Henriksen $T B$, et al. Surfactant need by gestation for very preterm babies initiated on early nasal CPAP: a Danish observational multicentre study of 6,628 infants born 2000-2013. Neonatology. 2017;111:331-6.

38. De Martino L, Yousef N, Ben-Ammar R, Raimondi F, Shankar-Aguilera S, De Luca D. Lung ultrasound score predicts surfactant need in extremely preterm neonates. Pediatrics. 2018;142(3). pii: e20180463. https://doi.org/10. 1542/peds.2018-0463.

39. Makri V, Hospes B, Stoll-Becker S, Borkhardt A, Gortner L. Polymorphisms of surfactant protein $B$ encoding gene: modifiers of the course of neonata respiratory distress syndrome? Eur J Pediatr. 2002;161:604-8.

40. De Luca D, Harrison DA, Peters MJ. 'Lumping or splitting' in paediatric acute respiratory distress syndrome (PARDS). Intensive Care Med. 2018:44:1548-50.

41. Bhatia R, Morley CJ, Argus B, Tingay DG, Donath S, Davis PG. The stable microbubble test for determining continuous positive airway pressure (CPAP) success in very preterm infants receiving nasal CPAP from birth. Neonatology. 2013;104:188-93. 